\title{
Desplazamiento forzado en adolescentes desde la experiencia límite según el modelo logoterapéutico*
}

Forced displacement of adolescents from limit experience according to the logotherapy model

Deslocamento forçado em adolescentes desde a experiência limite segundo o modelo logo terapêutico

Recibido el 30 de enero de 2015. Aceptado el 3 de mayo de 2015

Juliana Montoya Otálvaro**

Colombia

\section{Resumen}

> Para citar este artículo:

Montoya Otálvaro, Juliana

(2015). Desplazamiento forzado en adolescentes desde la experiencia límite según el modelo logoterapéutico. Ánfora, 22 (38), 135 -159. Universidad Autónoma de Manizales. ISSN 0121-6538
Objetivo: comprender el sentido de las circunstancias querodeanel desplazamientoforzadoenadolescentescomo una experiencia límite desde el modelo logoterapéutico. Metodología: se realizó una búsqueda de información, tanto en fuentes primarias como secundarias, a partir de tres categorías de análisis: situación de desplazamiento en Colombia, adolescente en situación de desplazamiento y planteamiento logoterapéutico ante situaciones límites. Se procedió a realizar una lectura crítica buscando la organización y estructuración de los datos. Resultados: se encontraron investigaciones que refieren causas y

\footnotetext{
*El artículo surge de la construcción del estado del arte de la investigación: "Sentido de vida en adolescentes desplazados por la violencia en Colombia".

** Doctora en psicología. Profesora e investigadora de la Fundación Universitaria María Cano. Colombia. Correo electrónico: julianaandreamontoyaotalvaro@fumc.edu.co y julianita_m17@hotmail.com
} 
consecuencias del desplazamiento forzado, especialmente en adolescentes, en relación con las implicaciones sociales y psicológicas. Se hallaron investigaciones recientes que analizan la capacidad resiliente de la población adolescente, identificando, en ello, factores protectores. De igual forma, se identificaron investigaciones desarrolladas

desde la Logoterapia, por la actitud que se toma frente a ciertas experiencias límites, lo que conlleva a analizar la situación de desplazamiento en adolescentes como una situación límite a la cual es posible encontrar o no sentido. Conclusiones: el adolescente desplazado, cuenta con elementos ontológicos que le permiten enfrentarse ante la posibilidad de encontrar un sentido. La Logoterapia afirma que el área de las actitudes es la más significativa, debido a que el hombre se enfrenta a situaciones adversas como el sufrimiento, la culpa o la muerte. Frente a dichas situaciones, como el desplazamiento, la actitud que asume el adolescente puede conducirlo a encontrarle sentido a su existencia aun en estos aspectos.

Palabras claves: Desplazamiento forzado, Adolescentes, Experiencia límite, Logoterapia

\section{Abstract}

Objective: to understand the sense of the circumstances that surround forced displacement of adolescents as a limit experience from the logotherapy model. Methodology: both primary and secondary sources were used to gather information about three analysis categories: displacement situation in Colombia, displacement situation of adolescents, and logotherapy approach in limit experiences. The organization and structure of data was based on a critical reading approach. Results: research referred to causes and consequences of forced displacement, especially of adolescents, in terms of social and psychological implications. Recent studies on the resilient capacity of adolescent population and the protective factors were also found. Likewise, other studies conducted from a logotherapy approach, due to the attitude to certain limit experiences, lead current research to analyze the situation of displacement of adolescents as a limit experience, which may or may not make sense. Conclusions: the displaced adolescents have ontological elements that allow them to confront the possibility of finding sense. Logotherapy states that the area of attitudes is the most significant since the man faces adverse situations such as suffering, guilt or death. When adolescents face situations such as displacement, their attitude in assuming the situation can make sense of their existence even within these circumstances.

Key words: Displacement, Adolescents, Limit experience, Logotherapy 


\section{Resumo}

Objetivo: compreender o sentido das circunstâncias que rodeiam o deslocamento forçado em adolescentes como uma experiência limite desde o modelo logo terapêutico. Metodologia: foi realizada uma busca de informação, tanto em fontes primárias quanto secundárias, a partir de três categorias de análise: situação de deslocamento na Colômbia, adolescente em situação de deslocamento e enfoque logo terapêutico ante situações limites. Procedeu-se a fazer uma leitura crítica procurando a organização e estruturação dos dados. Resultados: encontraram-se investigações que referem causas e consequências do deslocamento forçado, especialmente em adolescentes, em relação às implicações sociais e psicológicas. Encontraramse investigações recentes que analisam a capacidade de fortaleza da população adolescente, identificando, nisso, fatores protetores. Similarmente, identificaram-se investigações desenvolvidas a partir da Logo-terapia, pela atitude que se tem à frente de certas experiências limites, o que acarreta a analisar a situação de deslocamento em adolescentes como uma situação limite à qual é possível encontrar ou não sentido. Conclusões: o adolescente deslocado, conta com elementos ontológicos que the permitem lidar com a possibilidade de encontrar um sentido. A Logo-terapia afirma que a área das atitudes é a mais significativa, devido a que o homem se enfrenta a situações adversas como o sofrimento, à culpa ou a morte. À frente de tais situações, como o deslocamento, a atitude que assume o adolescente pode conduzi-lo a encontrar sentido a sua existência ainda em estes aspectos.

Palavras-chave: Deslocamento forçado, Adolescentes, Experiência limite, Logo-terapia. 


\section{Introducción}

Para diciembre de 2012, la suma de víctimas de desplazamiento forzado en Colombia ascendía a 5’701.996 desde 1985, de acuerdo con las estimaciones históricas de la Consultoría para los Derechos Humanos y el Desplazamiento (CODHES, 2013).

Este fenómeno, consecuencia del conflicto armado, es una realidad que se repite a diario, de tal manera que en el año 2007, según el informe de la oficina del Alto Comisionado de las Naciones Unidas para los Refugiados (ACNUR), este país ocupaba el segundo lugar en el mundo en desplazamiento interno, pues cerca del $8 \%$ del total de la población se encontraba en tal situación y se estima que esta cifra aumenta día tras día (Summerfield, 2000).

Al hacer referencia al desplazamiento, éste se entiende como aquel fenómeno en el cual una persona es sacada por la fuerza del lugar donde se encuentra residiendo. Se hace importante reconocer la definición de desplazado que presenta el Artículo 1 de la Ley 387 de 1997:

Es desplazada toda persona que se ha visto forzada a migrar dentro del territorio nacional abandonando su localidad de residencia o actividades económicas habituales, porque su vida, su integridad física, su seguridad o libertad personales han sido vulneradas o se encuentran directamente amenazadas, con ocasión de cualquiera de las siguientes situaciones: conflicto armado interno, disturbios y tensiones interiores, violencia generalizada, violaciones masivas de los derechos humanos, infracciones al derecho internacional humanitario $\mathrm{u}$ otras circunstancias emanadas de las situaciones anteriores que puedan alterar o alteren drásticamente el orden público (Congreso de Colombia, 1997, Art. 1).

En esta definición se observa que la decisión de dejar atrás su lugar de origen, sus tierras, los lazos establecidos, es forzada a través de la intimidación, a través de la violencia y a la amenaza de la vida propia y de personas cercanas.

Bernal (2009), en relación con el desplazamiento de personas, manifiesta que existen los siguientes tipos:

a. Según la causa: derivado de conflictos armados, intereses económicos, políticos, sociales, laborales, académicos, etc.;

b. Según el número de personas que se desplazan: individual, familiar y grupal o colectivo. 
c. Según la duración del desplazamiento: temporal o definitivo.

d. Según el lugar receptor: desplazamiento interno, que no sobrepasa las fronteras del país en donde se origina, y externo, que sí las sobrepasa.

Esta problemática es considerada como un acontecimiento estresor traumático que pone a prueba la estabilidad propia y la solidez del núcleo familiar y social. En ella prevalece el trauma psicológico y social que se refiere a un daño particular que es infligido a través de una circunstancia difícil o excepcional, que deja una impresión duradera en el subconsciente. Es un proceso complejo al que se enfrenta una persona, ya que deriva de un hecho que amenaza constantemente la supervivencia (Ramírez, Chávez y Molano, 2003).

Según la literatura internacional, los niños y adolescentes víctimas del conflicto armado o de situaciones límite, tienen altas tasas de compromiso psicológico. Se han reportado cifras hasta del 20\% de Trastorno por estrés postraumático en niños víctimas del conflicto en Uganda como el cuadro nosológico mental con mayor prevalencia dentro de esta población (Peltzer, 1999). La característica central en el adolescente y el desplazamiento es el cambio masivo de objetos externos e internos, que implican grandes pérdidas, ponen a prueba el psiquismo, reactualizan anteriores duelos y exigen modificar diferentes posiciones en la existencia.

El objetivo de la presente revisión bibliográfica es comprender el sentido de las circunstancias que rodean el desplazamiento forzado en adolescentes como una experiencia límite desde el modelo logoterapeutico. En la psicología existencial, se encuentra la logoterapia, entendiéndose por ésta la terapia mediante el logos que hace referencia al "sentido y significado", buscado por el hombre ante las circunstancias de la vida. El "logos" alude a la dimensión noética presentada por Viktor Frankl, fundador de la Logoterapia, como la dimensión espiritual. Por eso, la Logoterapia se refiere a una terapia por medio del sentido del significado (Luna, 2011).

A partir de lo que el adolescente desplazado ha vivenciado y significado, es importante para la logoterapia indagar cómo esta situación de desplazamiento le da al joven la posibilidad de encontrar o no un sentido de acuerdo con Frankl (1988) para quien el sufrimiento deja de ser, en cierto modo, sufrimiento en el momento en el que se encuentra un sentido y, por el contrario, la desesperación se produce por un sufrimiento vivido como sin sentido. 
La logoterapia toma de varios filósofos existencialistas, como Heidegeer y Kierkergaard, los conceptos de libertad y responsabilidad. La libertad implica una posibilidad para elegir. Está relacionada con el "ser y deber ser" y esto a su vez se relaciona con las metas y propósitos. Para la logoterapia es necesario un equilibrio entre lo que se es y lo que se debería ser. Este equilibrio evita caer en la frustración existencial. Esta libertad, a su vez debe ir acompañada del sentido de responsabilidad que guarda relación con el "deber ser". Esta responsabilidad se refiere a la capacidad que tiene el ser humano para responder ante las preguntas de la vida (Luna, 2011).

Así, el adolescente desplazado se encuentra ante innumerables preguntas sobre el "ser" y el "deber ser" de su situación. La identifica como una circunstancia límite que puede llevarle a diversas crisis, que se suman a las esperadas en esta etapa de la vida. Por lo anterior, es necesario indagar cómo esta situación de desplazamiento presenta al joven la posibilidad de encontrar o no un sentido, un significado a su existencia, brindando a la Logoterapia elementos de una intervención orientada a animar una mejor calidad de vida para la población juvenil.

El objetivo de la investigación -"Sentido de vida en adolescentes desplazados por la violencia en Colombia" - de la que hace parte esta Revisión estuvo orientado a indagar por el sentido de vida de adolescentes entre los 12 y 18 años que han sido desplazados por la violencia en Colombia. Los resultados este estudio corroboran los postulados de la Logoterapia frente a que sí es posible encontrar un sentido, a partir de la vivencia de una situación límite, potencializando los elementos positivos y de esta forma transformando los negativos. Así mismo, esta investigación le otorga a la psicología, y en especial a la Logoterapia, elementos de intervención para la población adolescente desplazada por la violencia en Colombia.

\section{Metodología}

Esta revisión bibliográfica se construyó como parte de la tesis doctoral "Sentido de vida en adolescentes desplazados por la violencia en Colombia". $\mathrm{Al}$ elaborar el planteamiento del problema se identificaron tres categorías de análisis fundamentales para guiar el trabajo investigativo.

Se partió, en un primer momento, de un estudio sobre el desplazamiento forzado en Colombia identificando su recorrido histórico y estableciendo sus causas y consecuencias. En un segundo momento, se hizo una observación del adolescente en situación de desplazamiento, identificando y relacionando los 
elementos característicos de este periodo juvenil; con ello se buscó una mayor comprensión del impacto de este fenómeno en esta etapa del desarrollo humano.

Para finalizar, se efectúa una revisión sobre los aportes de la Logoterapia específicamente en relación con las experiencias límites, para poder vislumbrar el desplazamiento forzado en adolescentes como experiencia límite.

Una vez establecidos los objetivos de la Revisión, se procedió a realizar la búsqueda bibliográfica a través de fuentes de información primaria y secundaria. Se recurrió además a las bases de datos google académico, proquest y scielo.

Los criterios de búsqueda fueron:

- Artículos sobre el desplazamiento forzado en Colombia

- Estudios sobre adolescentes desplazados por la violencia

- Artículos que analizaran el sustento Logoterapéutico frente a situaciones límites (muerte, culpa y sufrimiento)

Se realizó una evaluación de la información recopilada frente a su pertinencia, fiabilidad y validez (diseño y ejecución de las investigaciones) en relación con el problema abordado. Al seleccionar la información relevante, se procedió a realizar un análisis descriptivo de los datos presentados en las fuentes de información para presentarla de forma lógica y coherente en relación con las características descritas anteriormente de cada categoría de análisis establecida.

\section{Resultados}

Se encontró que, a diferencia de otros países en los que el desplazamiento surge a partir de un hecho de guerra concreto y específico, en Colombia el desplazamiento hay que mirarlo desde una continuidad histórica: desde la fundación de la República hasta el presente. Naranjo, Nieto, Jaramillo y González (2001) plantean que durante los procesos de colonización ocurridos en el siglo XIX se presentaron las guerras civiles ocasionadas por la persecución de aquellos que se consideraban como peligrosos para el poder dominante de la época. Por eso, poblaciones enteras se vieron obligadas a buscar refugio en regiones poco pobladas en donde el control institucional era débil o inexistente.

Así mismo, Kerr (2010) a través de un recorrido histórico reconoce cómo se ha ido desarrollando el desplazamiento forzado en Colombia. La primera fase de este 
fenómeno ocurrió en los años cincuenta del siglo XX durante el período de conflicto político conocido como "La Violencia”, que es visto como el precursor directo del conflicto actual, en los que unos dos millones de personas huyeron de sus hogares. La segunda fase del desplazamiento forzado tuvo lugar veinte años después, en los años setenta, cuando aumentaron los conflictos políticos a medida que la izquierda incrementaba su presencia y sus actividades y el gobierno colombiano respondía con represión. La fase actual empezó a mediados de los años ochenta cuando parte de la oligarquía del país creó las fuerzas paramilitares para erradicar a la guerrilla. Durante esta fase se empezó a afectar el campo colombiano.

Al analizar este recorrido histórico se puede observar cómo, en Colombia, para el desplazamiento se usan estrategias de terror empleadas para expulsar a la población y controlar territorios estratégicos: masacres, persecución y asesinatos selectivos de personas acusadas de ser auxiliadores de la guerrilla o de los paramilitares; tomas armadas de pequeñas poblaciones, retenes y control de acceso a víveres, prohibición de la circulación y de la práctica de determinadas actividades económicas o ejercicios profesionales, reclutamiento forzado de hombres y jóvenes para los diversos ejércitos, violación de mujeres, entre otros, son algunas de las situaciones que han "motivado" estos desplazamientos forzados (Villa, 2006).

A partir de lo anterior, se identificó la violencia como una de las variables que acompaña al proceso de desplazamiento. Kerr (2010) manifiesta que las violaciones a los derechos humanos que causa este fenómeno en la población civil incluyen: asesinatos, desapariciones forzadas, masacres o torturas. Molano (2001), afirma que "las personas no se desplazan por un acto de voluntariedad por el cual quieran entrar en devenir, más bien es la fuerza y la intimidación lo que los mueve" (citado por Castro y Jaramillo, 2014, p. 33). De ahí que este fenómeno no sólo se da de una forma forzosa, sino que se encuentra altamente relacionado con la intimidación y el miedo. De igual manera, Pecaut (1998) expresa que los recuerdos de estas violaciones son transmitidas de generación en generación; y el sentimiento del miedo transmitido es vivenciado como si hubiera sido parte de éste.

Reátiga (2013) a partir de testimonios sobre el desplazamiento concluyó que éste comienza con una vulneración de los derechos, en un primer lugar, en relación con el ámbito familiar seguido con el derrumbe del barrio, la escuela y la vereda. A esta tragedia se conoce como el "síndrome de la amenaza", mediante la cual se exige el desalojo del lugar, determinando un tiempo límite para llevarlo a cabo, pasando así al desplazamiento forzado. Bello (2004) expresa que las personas se enfrentan 
antes del desplazamiento a situaciones violentas, mediados por la desprotección y generando sentimientos de miedo, terror, impotencia y ansiedad y obligándolos, en la mayoría de los casos, a tomar la decisión de salir y dejar todo.

Una vez establecidas las posibles causas del desplazamiento, es importante reconocer las implicaciones que esta problemática ha generado en la población, identificando la pérdida de la tierra, la vivienda y los activos de los hogares. Esto ha ocasionado, a su vez, desempleo, marginación, deterioro en las condiciones de salud, inseguridad alimentaria, desarticulación social, empobrecimiento y, en general, el deterioro acelerado en las condiciones de vida en los municipios receptores (Ibáñez y Moya, 2006). Lo anterior conlleva al individuo a un desequilibrio físico, emocional, social, económico que pone a prueba sus estrategias de afrontamiento frente a su situación como desplazado.

Granada y Domínguez (2012) hacen referencia a los efectos del desplazamiento forzado en las familias, en donde se encuentra una desorganización de la cohesión familiar, poniendo en riesgo la unidad familiar, lo que lleva a los padres en medio de situaciones adversas a afrontar la crianza de sus hijos.

Falla, Chávez y Molano (2003) identificaron en sus investigaciones efectos psicosociales generados por el desplazamiento, a partir de lo cual este fenómeno puede ser considerado como un estresor traumático que pone a prueba la estabilidad personal y la solidez del núcleo familiar y social. Entre estos efectos del desplazamiento se identificó el trauma psicológico y social que se refiere a un daño particular infligido a una persona a través de una circunstancia difícil o excepcional y que deja una impresión duradera en el subconsciente. Es un proceso complejo al que se enfrenta una persona, ya que deriva de un hecho que amenaza constantemente la supervivencia. Cuando ocurre un evento traumático, el sistema nervioso central pierde la capacidad de controlar los efectos de desorganización que produce la experiencia y provoca un estado de desequilibrio. Se identificaron sucesos o condiciones que provocan reacciones físicas o psicológicas en una persona que ha sufrido dicho trauma como estrés, ansiedad, temor, depresión, pérdida de autoestima, paranoia, proceso de culpabilizar o culpabilizarse, deseo de venganza, asumir rol de víctima, empeoramiento de las condiciones de vida, desarraigo cultural, ruptura de redes sociales y afectivas, cambio en los roles de las familias, entre otras.

En relación con estos efectos psicosociales, Rodríguez, De la Torre y Miranda (2002) enuncian tres principales grupos de problemas psicosociales: 
- Desorden social, violencia y consumo de sustancias adictivas

- El miedo y la aflicción como consecuencia de los daños y pérdidas o por el temor a la recurrencia del problema

- Trastornos psicopatológicos o enfermedades psiquiátricas evidentes.

Entre estos problemas psicosociales se encuentra la pérdida como una de las características más representativas en esta población que lleva a la persona, en condición de desplazamiento, a enfrentar duelos de diferentes tipos, muchos de ellos debidos a la pérdida de un ser querido. De este tipo de duelos algunos no son elaborados y otros se realizan parcialmente; debido a las circunstancias especiales en los que se suceden, bloquean de manera especial las tendencias al adecuado funcionamiento mental y conductual (Valencia, 2008)

En relación con los efectos psicosociales de fenómeno estudiado aquí, se encuentran investigaciones que hacen énfasis de éstos sobre la población adolescente. En éstas, se evidencia en la adolescencia y el desplazamiento diferentes pérdidas que exigirán elaborar duelos en diferentes niveles. Aberastury y Knobel (1971) consideran que hay básicamente tres duelos: el duelo por el cuerpo infantil, el duelo de los padres infantiles, el duelo por la identidad infantil, que desencadena la exploración de una nueva, y que ocupa gran parte de la energía del joven a partir del momento en que se producen los cambios corporales.

Mier y Llamas (2003) encontraron que el adolescente desplazado se halla altamente vulnerable debido a la pérdida de continuidad de su identidad social, puesto que por su condición biológica y proceso evolutivo es una etapa crucial para la construcción de su identidad. Pero la situación de desplazamiento, situación de inestabilidad hace que dicha construcción se vea afectada significativamente.

Arias (2000, citado por Villamizar, 2011) manifiesta que en los desplazados menores de edad es frecuente encontrar historias de maltrato y pérdida de familiares. De igual forma, se observa un proceso de socialización enmarcado dentro del conflicto armado y de los simbolismos y valores de la guerra. Según los datos recogidos en el estudio de Bello, Mantilla, Mosquera y Camelo (2000) tres aspectos caracterizan la experiencia de la población infantil y juvenil en situación de desplazamiento:

1. Han vivido y visto la guerra; esto desestructura su mundo y las posibilidades de enfrentarlo debido todas estas experiencias de muerte, dolor y tragedia. 
2. Han sido socializados para sobrevivir en la guerra; esto crea desconfianza hacia los otros, produce una visión polarizada de la realidad entre lo bueno y lo malo.

3. Han tenido pérdidas abruptas y significativas, no sólo del hábitat natural, rural, medios de trabajo, sino sus seres queridos, amigos, vecinos.

A partir de estos aspectos que caracterizan la vivencia de desplazamiento en el adolescente, se identifica una serie de cambios que van influenciando el proceso de construcción de la identidad. Entre esos cambios se encuentran los culturales, cambios en la percepción de la realidad, cambios en la calidad de vida.

El concepto de identidad es crucial para el estudio de la adolescencia. Para Erikson (1974) este concepto significa una suerte de totalidad, un sentido de ser, que tiene sus raíces en la niñez y alcanza su mayor importancia en la adolescencia. En palabras de este autor "el joven, para experimentar la totalidad, debe sentir una continuidad progresiva en lo que se ha convertido al cabo de los largos años de niñez y lo que promete ser en el futuro anticipado” (Erikson, 1974, p. 87).

Al recordar las etapas planteadas por Erikson para describir el desarrollo del hombre, se hace referencia a las etapas 5 y 5 , las cuales describen las etapas del adolescente:

5. Identidad contra difusión de la identidad: nueva realidad corporal, búsqueda de una nueva identidad, superación de la confusión de identidad y confrontación de identidad en las relaciones heterosexuales.

6. Identidad contra aislamiento: se busca establecer relaciones íntimas, se presenta el aislamiento cuando en la intimidad se vivencia angustiosa, se puede ver el aislamiento como temor a esta.

Estas crisis están relacionadas con la infinidad de cambios característicos de este periodo. Este cambio lleva a la persona hacia la integración de los conocimientos en su vida, hacia la autonomía y hacia lo que llamaríamos el "sentimiento de responsabilidad" (Rice, 1998) y éste lo lleva a cuestionarse sobre lo ilógico del comportamiento de la sociedad en general y, particularmente, las personas que lo rodean. Comienza a darse cuenta de ciertas incongruencias entre las normas que se le establecen y los actos contradictorios de quienes las imponen, por lo que según Villanueva (1995) este universo de incongruencia produce en el adolescente desconcierto, frustración, rabia y lo hace interrogarse por el “ ¿Por qué?" Y al hacerse esta pregunta y al no encontrar respuestas se da cuenta de 
que piensa diferente a los demás, lo que lo lleva a darse cuenta plenamente de su propia existencia. Se podría decir que es un despertar existencial.

La crisis de esta fase consiste en hacer una elección compatible consigo mismo y con las oportunidades que ofrece la sociedad. Erikson (1974, citado por Barbieri, 2007) afirma que el adolescente tiene una existencia transitoria y que, por lo tanto, es existencialista por naturaleza. Esta etapa no constituye una afirmación sino una crisis normativa. La pregunta por el sentido de la vida, en este periodo, va de la mano de la pregunta por la identidad.

En la crisis de identidad se puede hacer referencia a dos aspectos: el clínico y el normativo. Las crisis de identidad patológicas tienen una propensión a autoperpetuarse, hay una creciente pérdida de energía defensiva y un aislamiento psicosocial, mientras que las crisis normativas son más transitorias. Así, emerge una abundancia de energía disponible que reactiva la ansiedad latente y provoca nuevos conflictos, pero que también fundamenta nuevas y amplias funciones del yo (Erikson, 1974).

A partir de lo anterior se podría decir que el adolescente desplazado llegaría a presentar una crisis de identidad normativa al atravesar una fase normal del desarrollo con un aumento de conflictividad, que podría a su vez presentar una crisis de identidad patológica teniendo en cuenta la traducción emocional de la violencia vivenciada en el desplazamiento. A lo anterior, Santacruz, Chams y Fernández (2006) expresan:

Tanto en la vida individual como en la colectiva, se desplaza entre el señalamiento de la psicopatía hasta la ruptura de los lazos sociales y el duelo por la pérdida de parientes, bienes y referentes históricos, así mismo, se menciona la irrupción de las lógicas de la guerra y la agresión en el discutir diario. Se destaca el acuerdo acerca de expresiones emocionales vinculadas con la violencia y el desplazamiento: miedo, desconfianza, ansiedad, temor, sufrimiento, estrés y otras más estructuradas como crisis, duelo y depresión (pp. 43-44).

Mier y Llamas (2003) concluyen que el adolescente desplazado se haya altamente vulnerable debido a la perdida de continuidad de su identidad social. Esto, porque debido a su condición biológica en una etapa crucial para la construcción de su identidad, la situación de desplazamiento, la situación de inestabilidad, hace que dicha construcción se vea afectada significativamente. 
Estas características de la crisis de identidad en los adolescentes desplazados, se encuentran reflejadas en los resultados de la investigación realizada por Barceló (2007) en la que se evidenciaron causas de morbilidad de los adolescentes desplazados prevaleciendo trastornos relacionados con la desorganización social como el consumo de alcohol, adicción a las drogas y hábito de fumar.

Al evaluar lo hallado sobre el desplazamiento forzado en población adolescente, se encuentran tres elementos característicos en la experiencia vivida: la muerte, el sufrimiento y la culpa, en donde los adolescentes se enfrentan al dolor de las pérdidas, el abandono, la imposibilidad de la elección, el fraccionamiento de la familia, el desarraigo y las carencias materiales son asumidas como una responsabilidad personal. La culpa aquí define en gran medida las relaciones que a partir de allí se construyen con sus propios familiares, con el sistema de pares, con los nuevos vecinos y la comunidad (Mesa de Trabajo de Bogotá sobre Desplazamiento Interno. Boletín No. 17 de 2006).

Estos tres elementos: muerte, sufrimiento y culpa, son abordados por la Logoterapia como la "triada trágica" en donde todo ser humano se enfrenta ante esta triada en el transcurso de su vida, y es precisamente la actitud que tome ante estos elementos lo que le permitirá encontrar significado a la experiencia límite. Al hacer referencia a las situaciones límites, Jaspers, (1973, p. 17, citado por González, 2002) las define como "situaciones de las que no podemos salir y que no podemos alterar”. A partir de esta definición, se puede ver el desplazamiento forzado como una experiencia límite, en donde el desplazado no puede modificar lo vivido pero si cuenta con la opción de asumir una actitud frente a su situación como desplazado.

Al describir esta triada trágica se encuentra que el sufrimiento permite al hombre transformarse e iniciar un proceso de maduración. Frankl (1994) plantea que "la adquisición de sufrimiento es un acto de configuración en la medida que el sufrimiento es ilimitado, pero también por el sólo hecho de estar mediado por valores actitudinales, que permiten encontrarle sentido al mismo sufrimiento" (p. 250). Esto relacionado con lo que postula la Logoterapia, no se puede cambiar la situación, pero sí se puede cambiar la actitud frente a ésta. La culpa, consecuencia de una decisión libre, encuentra su sentido en la medida en que las decisiones son irrevocables, pero no definitivas, según Sternig (citado por Guttmann, 1998). La muerte, el hombre es un ser ante la muerte y a ésta se encara desde la responsabilidad que se asume en la vida, desde su sentido y para qué. 
El adolescente en situación de desplazado se enfrentó y continúa enfrentándose a esta triada trágica: Por esto, tiene la capacidad de asumir una actitud en relación con las situaciones que se enfrenta a diario y que en ocasiones no se pueden cambiar. Lo anterior implica que todo ser humano se mueva entre la responsabilidad y la libertad de elegir.

Durante la adolescencia, la pregunta por el sentido de la vida se encuentra influenciada y mediada por la misma búsqueda de la identidad y la crisis que le acompaña. Es entonces cuando la persona se abre a otros espacios de socialización que dependerá en gran medida de la suficiente confianza dada durante los años anteriores. El conflicto básico que se presenta en esta etapa, es la lucha entre aquella parte que procura salir e independizarse y la que tiende a quedarse dentro del seno materno; esto representa en el adolescente ansiedad, conflicto interior y culpabilidad. El adolescente, necesita cortar para poder descubrir cuál es su sentido de vida. Este proceso implica alegrías, pero también sufrimiento y duelos necesarios para crecer (De Barberie, 2007).

Desde el análisis existencial y la logoterapia se plantean tres dimensiones del ser humano: una dimensión biológica, que hace referencia al cuerpo; una segunda dimensión psicológica que se refiere a la psique y una tercera dimensión espiritual (Noética), en la que se contempla la posibilidad de darle un sentido a la vida. Esta última, se convierte en la parte más esencial de cada ser humano y la que a su vez permite que cada uno resignifique la realidad dolorosa. Al hacer referencia a la dimensión noética, Frankl (1985) la define como el hilo conductor del análisis existencial y la logoterapia, porque contiene todas las capacidades del espíritu humano capaces de ser empleados por el individuo para contrarrestar la enfermedad y los traumas que la vida acarrea. Luna (2011) al hacer referencia al nivel espiritual (noético) lo describe como un nivel exclusivo del ser humano, en donde el espacio de la libertad es muy amplio; en donde éste puede tomar una actitud ante diferentes situaciones desafiando sus propios límites. Esta dimensión espiritual se diferencia de la dimensión psíquica en cuanto es la espiritual la que busca significados, un sentido a la realidad.

En la búsqueda del sentido, para la logoterapia los valores se transforman en la posibilidad de encontrar significados existenciales, lo cual permite eliminar la desesperación en situaciones de oscuridad existencial. Se hace referencia a tres tipos de valores: valores de creación, valores de experiencia, valores de actitud; estos últimos son los que permiten que el hombre pueda encontrar el máximo significado. Es a través de ellos que el adolescente desplazado puede encontrar sentido a su situación, a la experiencia vivida y acontecimientos 
desencadenantes. Es allí donde la actitud que toma frente a ese sufrimiento, culpa y muerte puede posibilitarlo a encontrar elementos en sí mismo y en su entorno que lo motiven a querer salir adelante y llevar a cabo su proyecto de vida. Frente a lo anterior García, Gallego y Pérez (2009) afirman que "El logro de sentido se asocia positivamente a percepción y vivencia de libertad; responsabilidad y autodeterminación; cumplimiento de metas vitales; visión positiva de la vida, del futuro y de sí mismo; y autorrealización” (p. 448).

Frankl (2003) manifiesta que un aspecto importante dentro de la Logoterapia es "La Voluntad de sentido" en la que si el ser humano posee el deseo de querer encontrar un sentido a su vida y la vive con plena felicidad, será capaz de superar el sufrimiento. Por el contrario, si el ser humano no es capaz de encontrar este sentido, a pesar de todo el bienestar que lo pueda rodear, podría estar inclinado a quitarse la vida.

El desplazamiento forzado, se convertiría en una experiencia a la cual es posible otorgarle un significado en la medida que el sentido de vida alude a la vida en general, pero siempre a situaciones concretas que cada individuo vive con sus propios recursos. Por esta razón, el sentido de la vida se presenta como la posibilidad de dar un significado y ayudar a encontrar un soporte interno a la existencia. Este deseo de significado es una necesidad específica no reducible a otras necesidades y está presente en mayor o menor grado en todos los seres humanos (Frankl, 1999). Además, dicho significado también puede servir de apoyo a la hora de afrontar situaciones adversas como lo sería el desplazamiento forzado a causa de la violencia, otorgándole al adolescente la posibilidad de darle sentido a esa experiencia o enfrentarse a un vacío existencial. El sentido es tener que realizar su propio ser. Este proceso significa reconocernos como naturalezaproceso, como proyecto y tarea y a ésta, como la búsqueda del sentido (Acevedo, 1998, p. 30).

En relación con la valoración del sentido de vida según las etapas del ciclo natural, para Yalom (1984) tal sentido debe contemplarse desde una perspectiva que permita apreciar su desarrollo en el tiempo, pues los tipos de significado cambian a lo largo de la existencia. Se produce un cambio de prioridades y de valores en el proceso de la vida, pues no aspira a conseguir lo mismo un niño, un adolescente, un adulto o un anciano. En cada periodo se desarrollan un tipo de valores.

Sumado a lo anterior, De Barbieri (2007) asevera que en la formación de la persona se distinguen tres tareas esenciales, manifestadas en cada ciclo vital: 
1. Conocerse: refiere a la propia interioridad y supone una tarea inacabable al intentar acceder a un dato cada vez más preciso y profundo ante el interrogante existencial “¿quién soy?”

2. Ubicarse "en el mundo": cubrir un espacio y asumir una posición a partir del conocimiento realista de sí mismo.

3. Proyectarse: movilizarse en una línea directriz de vida ante el interrogante “'hacia dónde debo-quiero-espero y puedo llegar?”

No obstante, es claro que no todo ser humano tiene un afán de encontrarle sentido a su vida o simplemente no lo encuentra. De esta manera, las personas se enfrentan ante una "crisis existencial" en la que se necesita un motivo más que material para ser feliz. Sin embargo, dicha crisis y sufrimiento es un camino hacia la madurez y hacia una posibilidad de encontrar sentido.

Lo planteado anteriormente, se relaciona con el concepto de resiliencia que ha sido trabajado recientemente por las áreas sociales extraído de la ingeniería, definida como la capacidad de una persona o grupo para seguir proyectándose en el futuro a pesar de acontecimientos desestabilizadores, de condiciones de vida difíciles y de traumas a veces graves.

Acevedo y Battafarano (2008) plantean que la resiliencia no puede ni debe ser vista solo como un medio adaptativo, con un criterio de homeostasis y de supervivencia individual o grupal; es una respuesta frente a situaciones externas; es la capacidad que tiene el ser humano para mantenerse integrado a pesar de la adversidad y buscar un desarrollo con sentido de acuerdo con su conciencia y con las metas de la propia cultura y/o como actor social frente a otras. Desde la Logoterapia puede entenderse el concepto resiliencia como la capacidad del ser humano de no estar sometido exclusivamente a las circunstancias.

La Logoterapia y la resiliencia tienen en común el reconocimiento de los factores protectores y la transformación de los comportamientos negativos en nuevas posibilidades (De Barbieri, 2002, citado por Noblejas y Rodríguez, 2005).

En la búsqueda de investigaciones con adolescentes desplazados se encontraron unas recientes enfocadas en la exploración de factores protectores y resilientes en esta población juvenil. En tales estudios, se busca ir más allá de las consecuencias negativas e identificar qué estrategias de afrontamiento se encuentran utilizando frente a su situación de desplazamiento. 
Por ejemplo, en la investigación realizada por Villamizar (2011) se encontró que la experiencia migratoria que han vivido los adolescentes colombianos participantes en el estudio ha favorecido a su proceso educativo, a las dinámicas de relacionamiento dentro de sus grupos familiares, a los niveles de participación juvenil y a sacarlos del entorno de la guerra. Así, en sus vidas el desplazamiento forzado por la violencia tiene en general una percepción positiva.

Llobet (2005) manifiesta que los niños y adolescentes en situaciones vulnerables, pueden presentar ciertas dificultades en el desarrollo psicosocial en función de las experiencias de adversidad que experimentan. Sin embargo, es esta misma adversidad lo que puede posibilitar experiencias promotoras del desarrollo, implementando estrategias positivas y resilientes impulsadas por la búsqueda de oportunidades y el deseo de superación. Lo anterior se evidencia en los resultados de la investigación realizada por Rodríguez (2006) en la que evalúa la eficacia de un programa de grupo estructurado en el desarrollo de estrategias de afrontamiento emocional, cognoscitivo y social para un grupo de adultos y adolescentes que desarrollaron estrés postraumático con posterioridad a una situación de desplazamiento en Colombia. Se encontró en las apreciaciones de los jóvenes, que a pesar de la pérdida al salir de su lugar de origen la ciudad representa una oportunidad. Así mismo, los resultados mostraron que la situación valorada en el pasado como amenazante, se convierte en una estrategia segura y saludable en el futuro, otorgando la posibilidad de afrontar situaciones difíciles en las que la autorregulación como proceso de autonomía, será de gran importancia.

En el estudio realizado en Colombia por González (2004) con familias desplazadas, se encontró en las entrevistas que independientemente del motivo del desplazamiento, estas personas reflexionan en torno a la reconstrucción de su proyecto de vida, asumen nuevas actitudes y desarrollan estrategias acorde con el nuevo sentido que le han dado a su vida.

De igual forma, en la investigación realizada por Morales (2009) se buscaba identificar las alteraciones psicológicas como consecuencia del desplazamiento forzado en Colombia en los adolescentes entre los 11 y 18 años víctimas de este proceso migratorio. Se encontró que la capacidad de resiliencia, la cual depende del entorno en gran medida, favorece la adaptación, por lo que no todos los adolescentes desplazados tienen procesos erráticos de aculturación al entorno.

Restrepo, Vinaccia y Quiceno (2011) realizaron una revisión teórica sobre los factores protectores relacionados en los procesos de resiliencia en donde 
encontraron factores tanto individuales como ambientales. Entre los individuales se resaltan las habilidades en la resolución de problemas y estrategias de afrontamiento, autoeficacia, autonomía, empatía, significado y propósito de la vida y el futuro y sentido del humor. Entre los factores ambientales se identifica la familia, la escuela y la comunidad. Lo hallado en esta revisión se relaciona con lo expuesto por Vanistendael (1995 y 2007, citado por Nuévalos, 2011) que reconoce, desde los aportes logoterapeuticos, cinco factores protectores que ayudan a la promoción de la resiliencia: la autoestima, el vínculo (afectivo y social), el sentido del humor, la creatividad, la red social y el sentido de pertenencia, la ideología personal y la voluntad de sentido.

Al evaluar estos factores, se puede evidenciar cómo el desplazamiento forzado puede considerarse en el adolescente una experiencia límite la cual, si bien lo enfrenta a factores de riesgo psicosociales, ella misma le brinda la opción de recurrir a la libertad de elegir la actitud frente a esta situación, reconociendo en él la capacidad de sobreponerse con sus propios recursos ante la situación adversa. Es por esto que la Logoterapia no se enfoca en el hombre enfermo; por el contrario, se enfoca en su fuerza, en la potencia espiritual de la persona de luchar ante la triada trágica, ante las crisis que tiene que vivir y a partir de ellas encontrar un sentido y autotrascender (Bazzi y Fizzoti, 1989, citado por Serralde, 2006).

\section{Conclusiones}

Al hacer referencia a las causas, los autores que realizan un recorrido histórico del desplazamiento en Colombia, llegan a la conclusión de que éstas se deben a una guerra por la adquisición de poder a nivel político y económico de diferentes grupos armados a través de los años, los cuales han recurrido al uso de la violencia y el terror como estrategias para alcanzar dicho poder.

Lo anterior ha generado en la población civil notables consecuencias físicas, psicológicas, sociales, políticas y económicas, a raíz de la pérdida de tierras, de masacres y asesinatos y constantes amenazas por la vida. A esto se suman los factores de riesgo con los que se encuentra el desplazado al llegar al lugar de acogida, donde encuentra desempleo, pocas condiciones de salud y vivienda y en algunos casos cierta discriminación social por su condición.

Específicamente en el adolescente desplazado, algunos autores que han evaluado estas consecuencias del ámbito físico, psicológico y social concluyen que éstas puedan afectar su calidad de vida. Teniendo en cuenta lo expuesto 
sobre la adolescencia, es de suma importancia evaluar el concepto de identidad en los jóvenes desplazados. Algunas investigaciones concluyen una interrupción en el proceso de adquisición de identidad social llevando a estos jóvenes a una situación de inestabilidad.

Al reconocer que la juventud es un periodo de grandes cambios, se suma al desplazamiento los cambios que trae consigo tal situación entre las que se encuentran los cambios culturales, de percepción y sobre su calidad de vida. De igual forma, algunas investigaciones concluyen que a raíz de las diferentes pérdidas que atraviesa el adolescente desplazado, éste atraviesa una serie de duelos que generan un desequilibrio emocional poniendo a prueba sus diferentes estrategias de afrontamiento.

Al analizar las teorías sobre el desarrollo de la identidad en el adolescente, varios autores concuerdan en la importancia del contexto y del ambiente, en la necesidad de ofrecer al joven espacios contenedores y experiencias que contribuyan a la vivencia de valores. Al analizar esto en contexto colombiano, específicamente en la experiencia del desplazamiento, se encuentra que, por el contrario, el adolescente se enfrenta a la violencia, el desarraigo y la adversidad, en donde se esperaría que esa construcción de su identidad se viera condicionada por su situación de desplazado. Sin embargo, es de gran importancia resaltar, que si bien se identifican investigaciones en donde se reconocen las implicaciones del desplazamiento en adolescentes, se reconocen investigaciones en donde se destaca a su vez la capacidad de resiliencia, de ir más allá, identificando en estos jóvenes una percepción positiva de su vivencia.

Este concepto de resiliencia se contextualizó a su vez a partir de los datos encontrados sobre logoterapia y situaciones límites. Nuévalos (2011) expresa "Estos dos modelos, la logoterapia y la resiliencia comparten una antropología que ofrece una visión positiva y esperanzadora de la persona como ser con valores, potencialidades y orientada desde su ser más profundo al crecimiento y a la promoción del bien propio y de los demás” (p. 170).

Respecto a las estrategias que presenta la Logoterapia para el afrontamiento ante situaciones límites, se encuentra la implementación de los elementos de salud que presenta el Logoterapeuta Acevedo (1994):

- Sentido de vida: plan de vida incluido en un proyecto familiar y social

- Posibilidad de escribir la propia historia vital en la familia, trabajo y la comunidad 
- Posibilidad de expresar lo no dicho en el dialogo y la reflexión

- Posibilidad de sentirse querido y de querer, privilegiando el encuentro con el otro

- Posibilidad de transformar y transformarse

- Posibilidad de comprometerse, de participar y de sentirse participando

- Posibilidad de establecer vínculos afectivos y de convivir.

Estos elementos se pueden identificar en algunas de las investigaciones que se han realizado con adolescentes desplazados, en donde se percibe su capacidad de narrar su experiencia, de dialogar sobre ésta. Son capaces de tomar lo vivido y transformarlo en un elemento de crecimiento, que les permite proyectarse a un mejor futuro. Y con el acompañamiento familiar y social busca, en su presente, una mayor inclusión dentro de la sociedad como jóvenes empoderados de su proyecto de vida.

En relación con la búsqueda de sentido ante las situaciones límites, específicamente ante el sufrimiento y la muerte, Frankl propone no una superación del límite sino un cambio de actitud puesto que, según la Logoterapia, la vida tiene un sentido que lo conserva bajo cualquier circunstancia en donde el sufrimiento y la muerte pueden transformarse en algo positivo en el encuentro con la adecuada actitud y disposición (Mèlich, 1994).

Al relacionar los datos encontrados sobre el desplazamiento, especialmente el adolescente desplazado con los elementos que presenta la Logoterapia en relación con la vivencia de situaciones límites, se concluye que el desplazamiento forzado en jóvenes se alcanzaría a considerar como una experiencia límite que los enfrenta ante la triada trágica: muerte, sufrimiento y culpa, conceptos abordados desde la Logoterapia. El ámbito de las actitudes es fundamental para encontrar un significado a su experiencia, en el que las situaciones adversas por las cuales atravesaron y atraviesan como desplazados, serán posibles elementos que le otorguen una mayor voluntad de sentido, recurriendo a valores de actitud, los cuales según la Logoterapia, permiten que el hombre pueda encontrar el máximo significado. Maurial (2009), en relación con estos valores, manifiesta que se pueden realizar en el momento en que el ser humano se encuentra ante situaciones límites y es allí donde la libertad tiene como finalidad asumir la responsabilidad de continuar viviendo aun sin la posibilidad de ejercer los valores creativos o vivenciales, el ser humano tiene la libertad de decidir la actitud que se asume ante aquella situación que ya no puede cambiar. 


\section{Referencias}

Aberastury, A. y Knobel, M.(1971). La adolescencia Normal. Buenos Aires, Argentina: Paidós

Acevedo, G. (1994). El modo humano de enfermar. Buenos Aires, Argentina: Fundación Argentina de Logoterapia "Viktor Frankl”

Acevedo, G. (1998). La búsqueda del sentido y su efecto terapéutico. Buenos Aires, Argentina: Fundación Argentina de Logoterapia "Viktor Frankl"

Acevedo, G. y Battafarano, M. (2008). Conciencia y Resiliencia. Buenos Aires, Argentina: Centro Viktor Frankl para la difusión de la Logoterapia

Barceló, R.(2007). Desplazamiento, salud y pobreza: Obstáculos para el desarrollo de los adolescentes más vulnerables de asentamientos marginales de Barranquilla (Colombia). Revista de Salud Uninorte, 23(2), 302-316

Bello, M. (2004). Identidad y desplazamiento forzado. Revista Aportes Andinos, (8), 2

Bello, M; Mantilla, L; Mosquera, C; y Camelo, E. (2000). Relatos de la violencia: impactos del desplazamiento forzado en la niñez y la juventud. Universidad Nacional de Colombia. Fundación Educativa Amor. Bogotá. Recuperado de: file:///C:/Documents\%20and\%20Settings/secretaria\%20CIDE/Escritorio/L-121-Bello_Martha-2000-153.pdf

Bernal, L. (2009). Atención en salud de familias desplazadas por la violencia: reflexiones desde la experiencia docente-asistencial. Univ. Med, 5o(2). Bogotá, Colombia

Castro, F. y Jaramillo, J. (2014). La conciliación en equidad ante el desplazamiento forzado. Reflexiones sobre sus posibilidades y límites. Revista de Derecho (42), 117-144

CODHES (2013). La crisis humanitaria en Colombia persiste. El pacífico en disputa. Informe de desplazamiento forzado en 2012. Documento CODHES $N^{o} 26$

Congreso de Colombia (1997). Ley 387 de 1997. Del desplazado y de la responsabilidad del Estado. Recuperado de: http://www.dhcolombia.info/ spip.php?article 1159 
De Barberie, A. (2007). Un sentido para todas las edades. Recuperado de: http:// www.logoforo.com/anm/templates/?a=282\&z=13

Erikson, E. (1974). Identidad, juventud y crisis. Buenos Aires, Argentina: Editorial Paidós, S.A.

Falla, U., Chávez, Y. y Molano, G. (2003). Desplazamiento forzado en Colombia. Tabula Rasa (1), 221-236. Bogotá, Colombia.

Frankl, V.(1985). La psicoterapia al alcance de todos. Barcelona, España: Editorial Herder

Frankl, V. (1988). El hombre en busca de sentido. Barcelona, España: Ed Herder

Frankl, V. (1994). El hombre doliente. Barcelona, España: Editorial Herder

Frankl, V. (1999). El hombre en busca de sentido último. Barcelona, España: Paidós

Frankl, V. (2003). La idea psicológica del hombre. Madrid, España: Editorial Rialp

García, J. Gallego, J. y Pérez, E. (2009) Sentido de la vida y desesperanza: un estudio empírico. Universitas Psychologica, 8(2) 447-454

González, E. (2002). Ensayo sobre las situaciones límite. NOUS, (6), 41-51. Recuperado de: de:http://scholar.google.com.co/ scholar?q=situacion +limite +logoterapia \&btnG $=\&$ hl $=$ en $\&$ as $\mathrm{sdt}=\mathrm{O} \%_{2} \mathrm{C} 5$.

González, C. (2004). Transformación y resiliencia en familias desplazadas por la violencia hacia Bogotá. Revista de Estudios Sociales, (18), 123-130

Guttman, D. (1998). Logoterapia para profesionales: Trabajo social significativo. Ed. Biblioteca de Psicología

Granada, P. y Domínguez, E. (2012). Las competencias parentales en contextos de desplazamiento forzado. Psicología Desde El Caribe, 29(2), 456-482

Ibañez A. y Moya A. (2006). ¿Cómo el desplazamiento forzado deteriora el bienestar de los hogares desplazados?: análisis y determinantes del bienestar en los municipios de recepción. Documento CEDE (26). ISSN 1657-7191 
Kerr, E. (2010). Desplazamiento forzado en Colombia, un crimen contra la humanidad. Boletín especial: Peace Brigades International Colombia. $N^{\circ} 14$

Llobet, V. (2005). La promoción de resiliencia con niños y adolescentes: entre la vulnerabilidad y la exclusión: Herramientas para la transformación. Noveduc Libros. Recuperado de: http://books.google.com.co/books?hl=en\&lr=\&id=ZCCphed_Ja4C\&oi=fnd\&pg $=$ PA $7 \& d q=$ desplazamiento $+y+$ resiliencia+en+adolescentes\&ots $=$ aPtn $2 q v y M r \& s i g=o U f H t g u G c G 6 e T w T S p-$

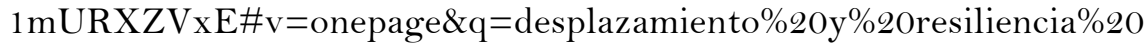
en\%20adolescentes $\& \mathrm{f}=$ false

Luna, A. (2011). Logoterapia: Un fenómeno Humanista Existencial Fenomenológico. Colombia: Editorial San Pablo

Maurial, I. (2009). Alfred Adler y Viktor Frankl: el discurso acerca del sentido de la vida. Avances En Psicología, 17(1), 35-58

Mesa de Trabajo de Bogotá sobre Desplazamiento Interno (2006). Boletín No. 17. Recuperado de: http://www.disaster-info.net/desplazados/informes/ mencoldes/17/boletin 17.pdf

Mier, E. y Llamas, Y. (2003). Identidad y dinámicas psicosociales en jóvenes desplazados en proceso de restablecimiento urbano, pertenecientes a la microcomunidad Revivir de los Campanos. Brocal, Revista de las Ciencias Humanas y de la Salud 3(2), 40-61

Mèlich, J. (1994). La construcción del sentido del sufrimiento y la muerte. Antropología filosófica y filosofía de la educación en Viktor E. Frankl. Enrahonar, (22), 93-103

Morales, J. (2009). Consecuencias psicosociales en los adolescentes en situación de desplazamiento. (Trabajo de postgrado en psiquiatría). Universidad Colegio Mayor de nuestra Señora del Rosario. Departamento de psiquiatría

Naranjo, G., Nieto, G., Jaramillo, A. y González, A. (2001). Desplazamiento forzado en Antioquia. Aproximaciones teóricas y metodológicas al desplazamiento de población en Colombia. Bogotá: Editorial Kimpres

Noblejas, M. y Rodríguez, M. (2005). Logoterapia, una ayuda para afrontar la adversidad. Aloma, revista de psicología (16), 105-116 
Nuévalos, C. (2011). La conducta antisocial desde una psicopedagogía positiva (una aproximación desde la logoterapia y la resiliencia). Revista Fuentes, $161-174$

Pecaut, D. (1998). El desplazamiento por la violencia en Colombia. Experiencias, análisis y posibles estrategias de atención en el Departamento de Antioquia. Memorias del Foro Internacional "Desplazados internos en Antioquia" Medellín, Colombia

Peltzer, K. (1999). Trauma and mental health problems of Sudanese refugees in Uganda. Central Africa Journal of Medicine 45(5), 110-140

Ramírez, U., Chávez, Y. y Molano, G. (2003). Desplazamiento forzado en Colombia. Análisis documental e informe de investigación en la Unidad de Atención Integral al desplazado (UAID) Bogotá. Revista tabula rasa. (1), 22 1-234

Reátiga, M. (2013). Ser adolescente hoy en Colombia. Algunas reflexiones desde la perspectiva psicodinámica. Barranquilla: Editorial Universidad del Norte

Restrepo, C., Vinaccia, S. y Quiceno, J. (2011). Resiliencia y depresión: un estudio exploratorio desde la calidad de vida en la adolescencia. Suma Psicológica, $18(2), 41-48$

Rice, F. P. (1998). Adolescencia: Desarrollo, relaciones y cultura. Madrid, España: Editorial Prentice

Rodríguez, M. (2006). Eficacia de un programa de grupo estructurado en estrategias de afrontamiento para DSPT en adultos y adolescentes en situación de desplazamiento. Universitas Psychologica, 5(2), 259-274

Rodríguez, J., De la Torre, A. y Miranda, C. (2002). La salud mental en situaciones de conflicto armado. Biomédica (22), 337-346.

Santacruz, C.; Chams, W. y Fernández, P. (2006). Colombia: violencia y salud mental. La opinión de la psiquiatría. Revista Colombiana de Psiquiatría, $35(1), 30-49$

Serralde, D. (2006). La adolescencia una etapa para el desarrollo de un proyecto de vida existencial. (Trabajo de grado Psicología). Universidad de la Sabana. Recuperado de: http://intellectum.unisabana.edu.co:8080/jspui/ bitstream/10818/4542/1/131004.pdf 
Summerfield D. (2000). Conflict and health: war and mental health: a brief overview. British Medical Journal xx. Recuperado de: http://www.ncbi. nlm.nih.gov/pmc/articles/PMC1118225/

Valencia, G. (2008). La psicoterapia breve humanista y su aplicación en el acompañamiento y la elaboración del duelo para personas en condición de desplazamiento forzado. Revista Médica de Risaralda, 14(2), 3-10

Villa, M. (2006). Desplazamiento forzado en Colombia. El miedo: un eje transversal del éxodo y de la lucha por la ciudadanía. Recuperado de: www.centromemoria. gov.c

Villamizar, C. (2011). Desarrollo de resiliencia en adolescentes en situación de desplazamiento forzado, en una comunidad educativa. (Trabajo de grado, inédito). Universidad Nacional de Colombia, Facultad de medicina, departamento de Pediatría. Bogotá. Recuperado de: http://www.bdigital. unal.edu.co/6277/1/598136-2011.pdf

Villanueva, M. (1995). Hacia un modelo integral de la personalidad. México: Editorial Manual Moderno

Yalom, I. D. (1984). Psicoterapia Existencial. Barcelona, España: Herder 